\title{
Achieving 'Good Health and Well-Being' for Women in Diverse Socio-Economic Neighbourhoods
}

\author{
Seyeon Lee ${ }^{1}$ \\ ${ }^{1}$ School of Design, Syracuse University, Syracuse, New York, Unite States of America \\ Correspondence: Seyeon Lee, School of Design, Syracuse University, Syracuse, New York, 13202, United States \\ of America. Tel: 1-315-443-2455. E-mail: slee80@syr.edu
}

Received: March 12, 2020

Accepted: April 2, $2020 \quad$ Online Published: May 26, 2020

doi:10.5539/jsd.v13n3p50

URL: https://doi.org/10.5539/jsd.v13n3p50

\begin{abstract}
Designing a place for women facing daily hardships often demands a distinct set of interventions that often bring unique challenges to designers. The Northside neighborhood is a poor and racially tensioned neighborhood with a lack of opportunity and social support. Many families in this neighborhood experiencing economic difficulties rising from such inequality and inadequate provision of opportunity, have raised concerns for no or limited access to basic necessities such as housing and healthcare. Particularly women in the Northside neighborhood raised their concerns about not a having socially and culturally safe environment in which they can engage in wellness activities (Stasya Erickson and Jonathan Logan, Personal Communication, June 18, 2018). Having daily challenges with childcare, financial hardship, accessibility, communication, cultural traditions that prevent them from taking care of their mental and physical wellness, these women do not have the luxury to concern about their health and wellbeing. Besides, conventional health facilities have been designed rather emphasizing the functional delivery of the space, which often lacked or unsuited to satisfy the psychological or social needs. This paper discusses the processes and results of three community design charrettes conducted to gather information on the need for women's fitness center with an aim to promote holistic means of wellness. The three design charrettes were organized with a) residents from the Northside community $(\mathrm{n}=15)$; b) local organizational stakeholders and community leaders $(\mathrm{n}=7)$, and $\mathrm{c})$ potential wellness center partners $(\mathrm{n}=2)$. In conjunction with the design charrettes, an alternative market study survey $(\mathrm{n}=108)$ was conducted to learn about people's opinions and views on the community's primary interests and the needs for the women-focused health system in the Northside neighborhood. Both charrettes and survey discovered that women's immense needs for childcare, sense of community, security, and privacy can be pursued through their participation to participate in the wellness activities. This research posits that design based on community's voices would help designers, educators, and policymakers to produce a realistic and impactful design that responds to the needs of women in diverse socio-economic neighborhoods.
\end{abstract}

Keywords: diverse socio economic neighborhood, women's health and wellness, good health and well-being

\section{Introduction}

Access to wellness often leads to improved fitness, physical health, and nutrition. It can also facilitate a sense of wellbeing and emotional, mental, intellectual, and psychological health (Hoeger et al., 2018; Ostgaard, 2006). Traditionally, health facilities have been designed to emphasize the functional delivery of the space, and often are lacking or unsuitable for meeting the psychological and social needs of users (Ulrich, 1991). Such conditions may result in negative consequences such as people not utilizing the space, implying a fundamental design failure. Therefore, all responsible parties - including designers, researchers, community stakeholders, and developers - should endeavor to critically identify user-centered ways of improving accessibility and engagement, creating physical surroundings that are functionally and psychologically supportive for all (Ulrich, 1991).

Designing a place for women with challenging backgrounds often demands a distinct set of interventions and thus brings unique challenges. Such an effort requires a broader perspective, one that encompasses diverse disciplines and practices, seeks ideas and views beyond the familiar scope, and looks at all dimensions of physical, cognitive, emotional, spiritual, and social life. Successful outcomes in this vein will improve the overall quality of life (Shepley \& Danko, 2017). While "quality of life" encompasses a broad scope have posited 
that ecology, economics, politics, and culture can all be used to access and affect this essential aspect of existence (Magee et al. 2012). One's wellbeing, gender, beliefs, education, and identity are all parts of the greater culture and means of achieving a high quality of life. However, as has been the case for many women in the Northside neighborhood, poverty and a lack of opportunity often prevent individuals from enhancing their quality of life (Hutchinson, 2018).

Syracuse's Northside neighborhood has historically been home to predominantly German and Italian working-class families. Once known for its lively community and financial stability (Gable, 2014), it was a vibrant neighborhood full of shops, restaurants, and active social organizations. Over the years, many of the original families left the neighborhood, and the area is now home to a diverse population of African Americans, as well as immigrants and refugees from South and Southeast Asia, the Caribbean, Africa, Eastern Europe, and the Middle East. There is also a growing population of young white professionals searching for new opportunities. Today, Syracuse's Northside is widely perceived as both a racially tense and economically disadvantaged community, with abandoned houses, boarded-up storefronts, unsafe neighborhoods, a general lack of opportunities, and pervasive poverty (Eisenstadt, 2018; Hutchinson, 2018). The good news is that although faced with many challenges, many areas are being rehabilitated, attracting a variety of organizations intent on improving economic opportunities and working to return the neighborhood to its once vibrant state (Moriarty, 2017).

Previous research completed by Northside UP, a program sponsored by the CenterState Corporation for Economic Opportunity and St. Joseph's Health discovered that most women in the Northside neighborhood face many obstacles to taking care of their personal wellbeing. Many residents voiced concerns about not having a socially and culturally safe environment to engage in wellness activities (Stasya Erickson and Jonathan Logan, Personal Communication, June 18, 2018). Women in Northside face challenges associated with childcare, financial hardships, accessibility, communication, and the influence of cultural traditions, just to name a few of the factors that prevent them from taking adequate care of their health and establishing a reasonable quality of life. It is difficult to break out of this cycle of daily hardships and continued impeded access to essential services eventually leads to generational issues that greatly impact the community's children and their parents' education and health, bringing lifelong effects (CNY Vitals, n.d.).

Since the adoption of the Beijing Declaration and Platform of Action in 1995, global partners along with the United Nations Commission on the Status of Women have expanded the agenda suggesting solutions to enhance women's health and wellness as human rights (UN Women, 2020). Making 25 years since the adoption, ensuring good health and well-being has become even more crucial for women in socio-economic neighborhoods (UN Women, 2020). Contributing to achieving sustainable development goals, exploring effective approaches to improving environmental quality would make a meaningful contribution to addressing women's wellness needs and may transform the quality of their lives. This research responds to the relevance of promoting physical activity and the general wellness of women in a local community by exploring multidisciplinary strategies for improving their physical environment.

This investigation will contribute to enhancing the overall body of knowledge regarding the design of user-centered wellness interiors for women. This research is meant to be a complementary resource, informing the future study, planning, design, and operation of women-focused spaces in diverse socioeconomic neighborhoods. This study will enhance the understanding of environmental design for women's wellness centers, with a particular focus on the diverse socioeconomic neighborhoods common in many parts of the world. The implications of the qualitative indicators presented here and their effectiveness will help designers, educators, and policymakers produce realistic designs and policies for improving the accessibility of women in challenged neighborhoods to health facilities and other community and educational services.

\section{Methods of Inquiry}

This study employed a multimethod process of inquiry to identify preliminary environmental design indicators $(i)$ useful in organizing women's fitness settings. The indicators were based on a review of the extant literature, three design charrettes, and a secondary-data analysis of a market study survey $(\mathrm{n}=108)$. 


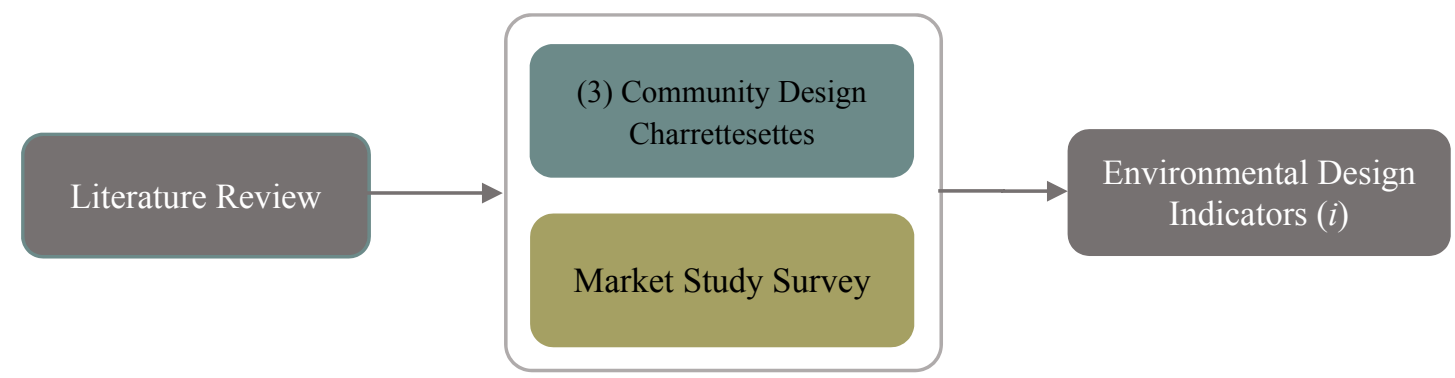

Figure 1. Methods of inquiry

\subsection{Community Design Charrettes}

Three community design charrettes were conducted, each a two-hour intense design collaboration and discussion session. The purpose of these design charrettes was to gather information on the need for a women's fitness center, with the goal of promoting holistic means of wellness. The three design charrettes were organized with: a) residents from the Northside community $(\mathrm{n}=15), \mathrm{b})$ local organization stakeholders and community leaders $(\mathrm{n}=$ $7)$, and c) potential wellness center partners $(n=2)$. Each charrette was organized around the topics of general, environmental, and spatial expectations for a future women-only fitness facility. Since the present research focuses on user-centered perspectives in designing women's wellness facilities, the third design charrette (i.e., the one with potential wellness center partners) is not discussed. Using a transcription of the other two design charrettes, distinctive words were extracted via Voyant Tools, an open-source online-based application that performs textual analysis (Voyant-tools.org, n.d.).

\subsection{Market Study Survey}

In conjunction with the design charrettes, NorthsideUP administered a market study survey $(n=108)$ to gather people's opinions and views on the community's primary interests and need for a women-focused health system in the Northside neighborhood. In addition, this survey allowed for an assessment of the community interest in and possible benefits from such a facility. As a secondary-data analysis of the survey results, only the findings relevant to female residents' interests in this type of facility and attraction to types of various exercises were used in this study.

\section{Findings}

Findings from both the community design charrettes and market study survey were used to gather onsite evidence from personal experiences and shared stories. Furthermore, a content analysis of user-centered design strategies identified in the community design charrettes and data compiled from the market study survey responses were used to identify environmental design indicators and offer a view of possible characteristics of a future women-focused health and wellness facility in the Northside community.

\subsection{Community Design Charrettes}

During the first community charrette conducted with residents of the Northside area, women from diverse ethnic and socioeconomic backgrounds gathered to voice their ideas and needs for a women-only fitness and wellness facility in the Northside neighborhood. Currently, there is no fitness space in this area that these women felt safe to access. Location, childcare, transportation, privacy, and cost were all important issues. The women explained that a place where they could solely focus on their health without having to be concerned about other members of their family, would be beneficial. Most importantly, an atmosphere that emphasized community, belonging, welcome-ness, care, and support was found to be essential.

A women-only facility would mean a safe and judgment-free environment where women could become healthier mothers to their children. A facility within their community that could be integrated into their lives and naturally foster positive peer pressure was of great interest to these residents. They were not interested in a women-only fitness facility located outside of their community. Within the Northside neighborhood, there would be more opportunities for friendship and help from one another. For deaf residents and people not yet fluent in the English language, transportation would also be an issue if the facility was located outside the community. Some residents explained that they had tried going to other fitness centers but felt culturally excluded.

Childcare was a main concern for these women. They needed to make sure their children were safe and cared for while they exercised. Other meanings of wellness in both education and consultation were needed to be included 
if the facility was to be a success. Accessibility regardless of age, culture, or level of ability needed to be reflected if a holistic design for the fitness center was to be achieved. The Northside neighborhood holds special meaning for its residents, and a place that would support community events would bolster their sense of connection.

Visibility, openness, enclosure, separation, and privacy were all significant and sometimes contradictory factors to these women because of their diverse backgrounds. No one should be able to look inside the facility from the street. They did not want to be seen by outsiders, regardless of who they were. They wanted to escape their surroundings and the people who occupied them when they made use of the facility. These women wanted to strengthen their community while inside this space. Even those meeting for the first time at the charrette seemed immediately comfortable with one another. The conclusion was that high windows would be preferred, allowing for natural lighting but inhibiting visibility.

Due to the current nature of the neighborhood, layers of security measures were also preferred. Many of the women lacked language skills. Thus, the residents strongly supported language-free wayfinding for easy access and communication. This would also assist with supporting people with disabilities. Residents hoped the facility would also support wellness-related amenities such as nutrition, medical checkups, women-centered education, nursing, counseling, and even a lounge space that would allow for social and support activities. A space for culturally competent support groups to hold meetings was also discussed as a means of better sustaining the facility.

Table 1. Distinctive words obtained from community design charrettes

\begin{tabular}{c}
\hline Distinctive Words* \\
\hline Children \\
Community \\
Fitness \\
Care \\
Childcare \\
Exercise \\
Privacy/Private \\
Welcoming \\
Access
\end{tabular}

Note. Common verbs, adjectives, and words such as "women". "space", "place", "center", and "gy," were eliminated from the textual analysis.

During the second community design charrette, the local organization leaders, stakeholders, researchers, and community partners shared perspectives that were different and yet in some ways similar to those expressed in the first design charrette. The participants associated the women-only fitness facility with words like "exercise," "community," "social," "inclusive," and "health." Based on the history and demographics of the Northside neighborhood, there were many community-organizing spaces that offered services to improve the health and wellness of residents. They were interested in this new space as a means of enhancing resources and access to wellness, and as well as a way of addressing equality in the community at large. Not only physical wellness, this center would also reduce social isolation by providing a safe space in which female residents could exercise. However, there were various concerns, such as women-only needs, accessibility for those with disabilities, childcare, and more. The space could potentially provide a comprehensive opportunity to meet the health and wellness requirements of women in the neighborhood.

The participants envisioned this facility as supporting general wellness and not solely focusing on physical fitness, though that was also important. It was essential that the space make people feel included and comfortable the moment they walked in off the street. Therefore, it needed to represent the culturally diverse neighborhood outside. Ultimately, the facility had to cultivate a culturally sensitive environment for women of a variety of socioeconomic backgrounds, ages, and ethnicities. 


\subsection{Market Study Survey}

A community survey entitled "Northside Wellness Project" was distributed to the residents of the Northside neighborhood for approximately 30 days, from August to September 2018. Out of the study's five domains personal demographics, the need for a wellness center, program desires, barriers to access, and financial sustainability - only the questions relevant to a user-centered perspective on designing a women-only fitness facility were evaluated for the present research. Due to the major demographics of the neighborhood, the survey was translated into six languages: Spanish, French, Vietnamese, Arabic, Nepali, and Swahili. Only 4\% of the distributed surveys were returned, resulting in a total response of 108 ; however, included in these were many incomplete responses.

A total of $33 \%$ of the participants responded that they were interested in a women-only fitness center, and $36 \%$ indicated that its location within the community and it being women-only would increase their level of interest (see Figure 2). Elyer et al. (2003) stated that although women in the neighborhood were aware of a number of places to exercise, the locations were not convenient, financially reasonable, or emotionally comfortable enough for them to actually access. Choitz et al. (2010) argued that a fitness facility within the community would be more attractive to community residents, as the space would already be a part of their lives. They would have a greater stake in maintaining it as a clean, safe, and welcoming environment. More importantly, it would improve accessibility and increase participation by women living in the community. The survey revealed that $50 \%$ of the female residents walked to their daily destinations; walking was their primary means of transportation.

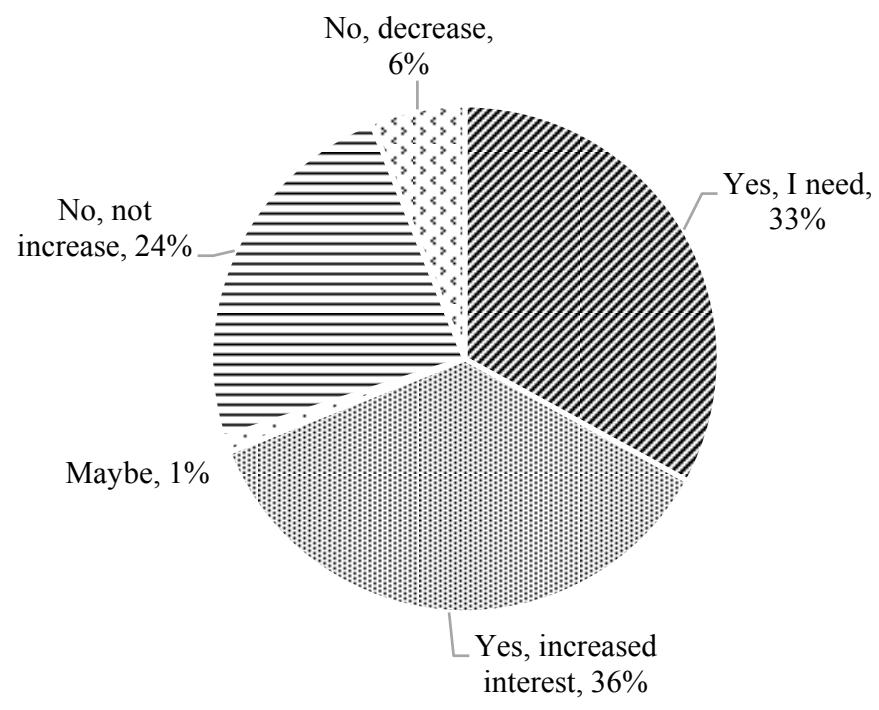

Figure 2. Interest in a women-only wellness center $(n=100)$

For women expressing interest in a women-only facility, the availability of onsite childcare was found to be either essential or at least a feature that would increase their interest in attending the facility (see Figure 3). 


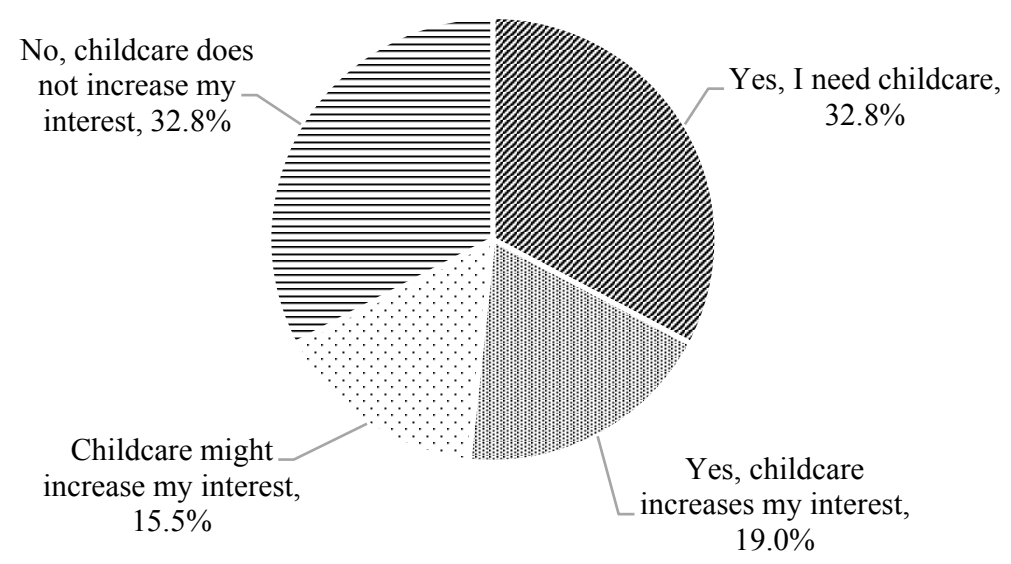

Figure 3. Interest in a women-only wellness center with childcare available $(\mathrm{n}=58)$

A total of $55.44 \%$ of female residents surveyed $(n=101)$ indicated that they would attend the facility two to four times per week. Group exercises such as dance, yoga, Zumba, and Pilates were most favored by the participants. Other types of exercises that could be done by themselves and without any time commitment (e.g., walking, running, using steppers or elliptical equipment) were also desirable (see Figure 4). Some indicated that swimming would be favored, but based on the feasibility of the facility, swimming or any other water-related exercises were not considered.

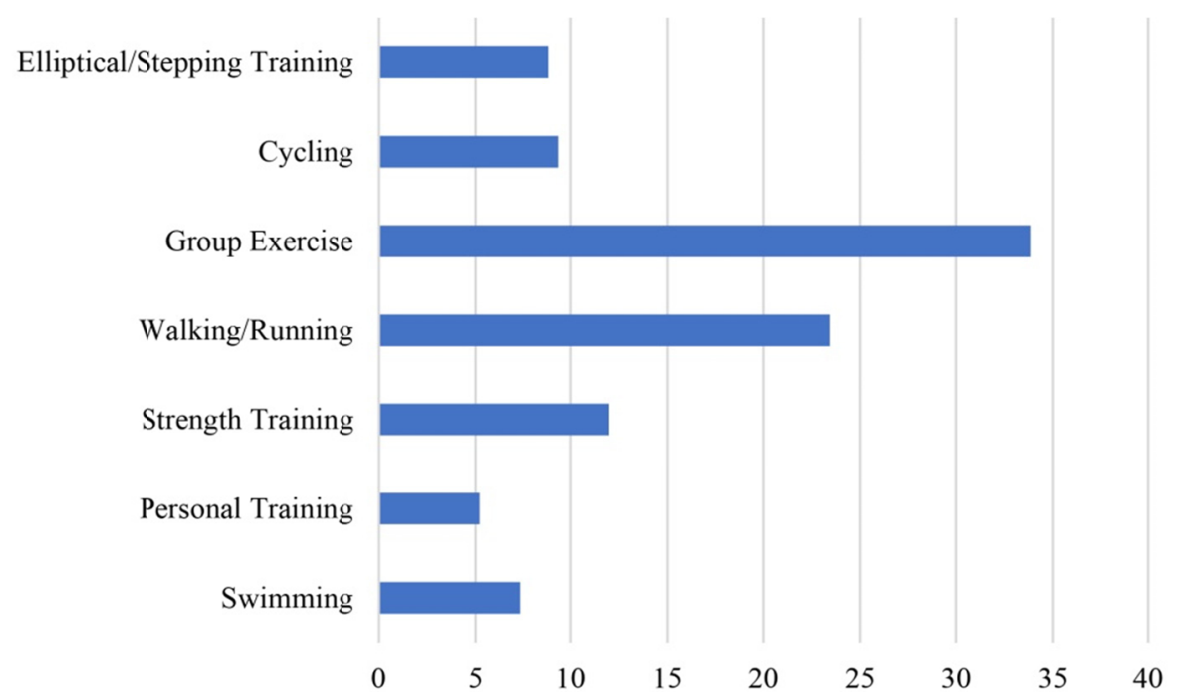

Figure 4. Desirable exercise programs and equipment $(n=56)$

\section{Five (5) User Centered Environmental Design Indicators}

The evolutionary quality of this research effort and its intended process did not lend itself to a level of scientific rigor that would be optimal in creating, testing, and validating user-centered environmental design indicators $(i)$ generalizable to designing women-only wellness facilities in other diverse socioeconomic neighborhoods. However, the list of indicators included here can potentially serve as a step towards developing a systematic strategy for designing women-focused wellness centers. This set of environmental design indicators includes: community, safety, sustainability, comfort, and accessibility. Not only these terms are interconnected, but also affect one another (see Figure 5). 


\subsection{Community: Culture, Background, Socialization, and a Sense of Community}

This study posits that community-based collaboration in the design process is crucial to securing the success of the project. Jemner and Stokols (2000, p2) asserted that wellness permeates "through all ... social, environmental, and other activities of populations." Similarly, promoting wellness means pursuing a change in lifestyle in terms of both behavior and living conditions. In the Northside neighborhood, community-based efforts, multicultural pride, and the reclamation of traditional approaches to health and wellness are regularly being promoted. The preservation of native languages and cultural traditions has contributed to the sense of community visible there today. Additionally, as each resident seeks a place in their multicultural society, the nature of their differences creates unique issues in terms of human services, including health and wellness (Weaver, 1998).

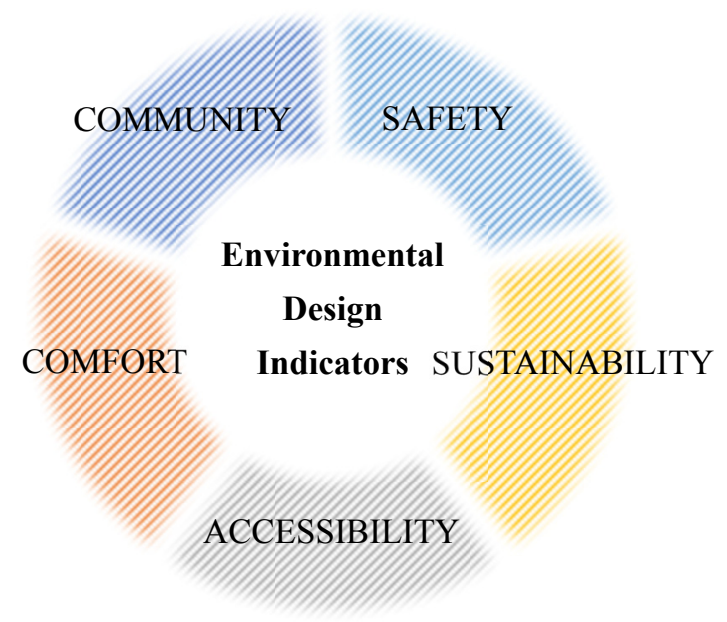

Figure 5. User-centered environmental design indicators

During the design charrette discussions, residents contributed their design ideas to the potential facility location. Insights into pedestrian traffic, parking, the history of residents and businesses adjacent to the potential site, views to avoid, and more were obtained from residents and local community leaders who knew the neighborhood to a level higher than any researcher could hope to achieve. For these reasons, understanding the needs of the community and its residents could not be separated from the broader discussion of the design for the wellness facility.

\subsection{Accessibility: Transportation, Location within the Community, and Language-Free Wayfinding}

The convenience of accessibility is essential for a wellness facility (Eyler et al. 2003). Ease of accessibility increases participation; this, in turn, financially sustains the facility, further improving economic growth in the surrounding area. The market study survey revealed that the facility being accessible by bus or walking would make access to the wellness center easier for women in the neighborhood. Moreover, this would have an intergenerational impact on mothers' and children's health. Accessing onsite childcare would allow mothers to leave their children to exercise; they could be temporarily separated from their children and thus left alone to concentrate on their health. With respect to cultural diversity and disabled women in the neighborhood, language-free support for navigating the facility and design interventions for communicating with others would make the facility more approachable and user-friendly. Linking these concerns regarding a broad level of accessibility would result in positive long-term experiences, securing holistic means of safety for these women.

\subsection{Cultural Safety, Privacy, Protection, and Care}

Cultural safety, as defined by Smye and Browne (2013), involves recognition of the social, economic, and political differences of groups within a given society. There are second and third generation immigrants from European countries, refugee families from various Asian and African nations, and young professionals, all living in proximity (CNY Vitals, n.d.). Nothing in the environment of the wellness center should threaten women or prevent them from participating in wellness activities. The results of the design charrettes revealed that many women were concerned about their privacy and protection, especially if there are any possibility that men might see them exercising. For example, many women in the neighborhood wear hijabs when outside; they would take 
off these hijabs only if they felt they were in a private and protected environment, and there was no risk of men seeing them. Physical and mental safety and protection can be emphasized in the furnishings and ambiance of the environment (Platt et al., 2017), directly affecting the design of an environment that inspires comfort.

\subsection{Comfort}

Research has suggested that a welcoming, well-lit, legible, home-like, non-institutional, and flexible environment most represents a comfort-based design strategy (Platt et al., 2017). Creating a comfortable environment for women, one in which they are free to focus on their wellness, is essential to ensuring the success of the facility. Studies have shown that social support and interpersonal interactions have a positive influence on health outcomes, and people talking to one another daily is reported to have profound effects on mental health. Comfort means being surrounded by others of a similar age and background, those who share a common generational point of reference (Gallagher-Thompson et al., 2017; Valadez et al., 2006). As mentioned above, "welcoming" was one of the distinctive words appearing in the discussion, and lighting, views, colors, distribution of spaces (both workout spaces and social gathering areas), furnishings, and interior furnishings are all factors contributing to the creation of a comfortable environment for the women of the Northside neighborhood.

\subsection{Sustainability: Education, Socialization, and Multiple Functions}

The primary purpose of the facility is to facilitate health and wellness activities in a safe and comfortable environment that is functionally and psychologically supportive of women's access to wellness (Ulrich, 1991). Most importantly, activities must be self-sustainable. Therefore, they should continuously evolve and always be geared toward improving the wellness of the women in the Northside neighborhood.

Discussions during the design charrette session with local stakeholders and community leaders suggested that there are many local organizations separately pursuing efforts to promote various levels of community health services. Representing several different perspectives on medicine and care, educational sessions on topics of general health care, medical insurance, senior care, pregnancy and contraception, nutrition, and rehabilitation of alcoholics are all currently being held in separate locations within the community. It was voiced in the discussion that this women's wellness facility could serve as a central location for health and wellness resources that impact the community. "Accessibility," "safety," and "comfort" within the "community" would make the facility an optimal location for local women to reach out, and also give the facility the opportunity to "sustain" itself via a long-term community business model and make a positive contribution to the community.

\section{Discussion}

The purpose of this research was to create organizational and environmental design indicators for user-centered design and describe systematic approaches that stakeholders might consider when designing and operating women-only wellness facilities servicing diverse socioeconomic neighborhoods. One of the challenges for developers, facility designers, and organizations is that there are very few comprehensive studies and guidelines available for improving access to wellness for women in lower socioeconomic and racial/ethnic minority groups. In addition, every community is unique; therefore, residents and design teams must collaborate to achieve positive outcomes and support user needs.

The environmental design indicators presented here are based on the Northside neighborhood in Syracuse, New York. Thus, they are to be considered as a reference, informing the future planning and design of additional women-only fitness facilities. These indicators should be used to inspire discussion and further develop comprehensive guidelines for new approaches to user-centered wellness facilities. As a robust list of environmental design indicators, this user-centered collection of design considerations focusing on community, accessibility, safety, comfort, and sustainability are meant to be a preliminary facilitator for those seeking to inspire a holistic means of wellness.

Future research should examine and test these environmental design indicators, exploring how they relate to other women-only facilities and suggesting improvements that might increase the potential for successful outcomes of user-centered goals. This study focused on a wellness setting for women in a diverse socioeconomic neighborhood. Each neighborhood carries its own site-specific characteristics and qualities; therefore, there are limitations to generalizing the research findings presented here to other women-only facilities and organizations.

\section{References}

Choitz, P., Johnson, M. P., Berhane, Z., Lefever, G., Anderson, J. K., \& Eiser, A. R. (2010, February 18). Urban Fitness Centers: Removing Barriers to Promote Exercise in Underserved Communities. Journal of Health Care for the Poor and Underserved, 21(1), 221-28. https://doi.org/10.1353/hpu.0.0239 
CNYVitals. (n.d). Poverty. CNY Vitals. Retrieved from https://cnyvitals.org/poverty/

Dew, B. J., \& Newton, K. S. (2005). Gender, sexual orientation, and wellness: Research implications. In J. E. Myers, \& T. J. Sweeney (Eds.), Counseling for wellness: Theory, research, and practice (pp. 127-138). Alexandria, VA: American Counseling Association.

Eisenstadt, M. (2018, June 13). 'Poverty has a women's face': New CNY website examines local poverty, education data. Syracuse.com. Retrieved from https://www.syracuse.com/news/index.ssf/2018/06/poverty_has_a_womans_face_new_cny_website_exami nes_local_poverty_education_data.html

Eyler, A. A., Matson-Koffman, D., Young, R. D., Wilcox, S., Wilbur, J., Thompson, J. L., ... Evenson, K. R. (2003, October 1). Quantitative Study of Correlates of Physical Activity in Women from Diverse Racial/Ethnic Groups: The Women's Cardiovascular Health Network Project Summary and Conclusions. American Journal of Preventive Medicine, Physical Activity in Women from Diverse Racial/Ethnic Groups: Environmental, Policy, and Cultural Factors, 25(3), Supplement 1, 93-103. https://doi.org/10.1016/S0749-3797(03)00170-3

Gable, M. (2014, December 28). Syracuse neighborhoods: Northside. Syracuse.com. Retrieved from https://www.syracuse.com/living/2014/12/syracuse_neighborhoods_northside.html

Gallagher-Thompson, D., Tazeau, Y. N., Basilio, L., Hansen, H., Polich, T., Menendez, A., \& Villa, M. L. (1997). The relationship of dimensions of acculturation to self-reported depression in older, Mexican-American women. Journal of Clinical Geropsychology.

Hoeger, W. W., Hoeger, S. A., Hoeger, C. I., \& Fawson, A. L. (2018). Lifetime of Physical Fitness and Wellness. Cengage Learning.

Hutchinson, S. (2018). Music and Food in Multicultural Syracuse: Project Report. Voices: The Journal of New York Folklore, 44(1-4), 46.

Jamner, M. S., \& Stokols, D. (Eds.). (2000). Promoting human wellness: New frontiers for research, practice, and policy. Univ of California Press.

Magee, L., Scerri, A., \& James, P. (2012). Measuring social sustainability: A community-centred approach. Applied Research in Quality of Life, 7(3), 239-261. https://doi.org/10.1007/s11482-012-9166-x

Moriarty, R. (2017). Central New York gets $\$ 86$ million from state for development projects. Syracuse.com. Retrieved from https://www.syracuse.com/news/2017/12/central_new_york_gets_86_million_from_state_for_development _projects.html

Ostgaard, G. D. (2006). For "Women Only": Understanding the Cultural Space of a Women's Gym through Feminist Geography (Doctoral dissertation, Bowling Green State University).

Platt, L. S., Bosch, S. J., \& Kim, D. (2017). Toward a Framework for Designing Person - Centered Mental Health Interiors for Veterans. Journal of Interior Design, 42(2), 27-48. https://doi.org/10.1111/joid.12095

Shepley, M. M., \& Danko, S. (2017). Design as Healing: The Next Generation of Research - Informed Practice. Journal of Interior Design, 42(1), 5-7. https://doi.org/10.1111/joid.12090

Smye, V., \& Browne, A. (2002). 'Cultural safety' and the analysis of health policy affecting aboriginal people. Nurse Researcher (through 2013), 9(3), 42-56. https://doi.org/10.7748/nr2002.04.9.3.42.c6188

Ulrich, R. S. (1991). Effects of interior design on wellness: theory and recent scientific research. Journal of health care interior design, 3(1), 97-109.

UN Women. (2020). United Nations Entity for Gender Equality and the Empowerment of Women (UN Women). Institutional Report.

United Nations. (1999). Status of Women Commission Concludes Discussion of Women and Health, and Follow-up to Beijing Conference. Meetings Coverage and Press Releases. WOM/1105. March 3, 1999. Retrieved from https://www.un.org/press/en/1999/19990303.wom1105.html

Valadez, A. A., Lumadue, C., Gutierrez, B., \& de Vries-Kell, S. (2006). Las Comadres and adult day care centers: The perceived impact of socialization on mental wellness. Journal of Aging Studies, 20(1), 39-53. https://doi.org/10.1016/j.jaging.2005.02.003

Voyant Tools. (n.d.). “About.” voyant-tools.org. 
Weaver, H. N. (1998). Indigenous people in a multicultural society: Unique issues for human services. Social Work, 43(3), 203-211. https://doi.org/10.1093/sw/43.3.203

\section{Copyrights}

Copyright for this article is retained by the author(s), with first publication rights granted to the journal.

This is an open-access article distributed under the terms and conditions of the Creative Commons Attribution license (http://creativecommons.org/licenses/by/4.0/). 